\title{
A QUANTITATIVE UNDERSTANDING OF TRANSIENT BUBBLE GROWTH IN MICROCHANNELS USING $\mu$ PIV
}

\author{
Evelyn N. Wang, Shankar Devasenathipathy, Carlos H. Hidrovo, David. W. Fogg, Jae-Mo Koo, \\ Juan G. Santiago, Kenneth E. Goodson, Thomas W. Kenny \\ Stanford University, Departments of Mechanical Engineering \\ Stanford, CA 94305
}

\begin{abstract}
Various fluidic MEMS structures require a better fundamental understanding of microscale bubble growth dynamics. Much of the work in this area has been qualitative observations of bubble formation and growth. This paper presents an approach to obtain quantitative estimates of forces acting on bubbles by obtaining liquid velocity fields using micron-resolution particle image velocimetry ( $\mu$ PIV). These velocity fields aid in determining parameters such as viscous stresses acting on the bubble, vapor and local liquid pressure, and bubble departure criteria. The ability to obtain these velocity fields in a transient bubble growth process is demonstrated in a microfabricated silicon channel with an integrated heater. These measurements offer a step towards understanding and developing accurate models for MEMS structures associated with the presence of bubbles.
\end{abstract}

\section{INTRODUCTION}

Bubbles are prevalent in several different MEMS devices. Examples include thermally-actuated pumps and valves [1-3] and two-phase microchannel heat sinks for IC cooling applications [4-6]. However, little is understood about the bubble formation and growth process in these microscale structures. A robust understanding of these phenomena is needed in order to optimize the performance of these MEMS devices.

This paper presents an experimental methodology which can potentially determine forces on growing vapor bubbles in a microchannel. By acquiring the liquid velocity field in a region near the bubble using micron-resolution particle image velocimetry ( $\mu$ PIV), important parameters such as viscous stresses, vapor and local liquid pressure, and bubble departure may be assessed. This important information will aid in a better fundamental understanding of bubble actuated devices, and boiling in microchannel heat sinks; and the methodology be extended to other MEMS structures and applications. In this paper, we demonstrate this approach for the first time in a heated microchannel system during heterogeneous nucleation and boiling for the future development of a robust two-phase heat sink model and design.

\section{EXPERIMENTAL APPROACH}

The $\mu$ PIV setup consists of an epi-fluorescent microscope (Nikon TE300) with a mercury lamp illumination source as shown in Figure 1. Imaging is achieved using a $20 \mathrm{X}$ objective lens $(\mathrm{NA}=0.75)$ and a 12-bit resolution interline cooled CCD camera (Roper Scientific CoolSnap HQ). A constant hydrodynamic head in a graduated cylinder generates flow through the microchannel test structure. Deionized water is seeded with $700 \mathrm{~nm}$ fluorescent polystyrene particles (Duke Scientific) at a particle density of $0.025 \%$. A concentration approximately half of the critical micelle concentration of Triton-X (Sigma) nonionic surfactant is used to reduce particle adsorption to the channel walls.
The liquid velocity fields surrounding a growing bubble are obtained using $\mu$ PIV by cross-correlating image pairs in designated interrogation regions. The PIV algorithm used in these experiments was developed by Meinhart et al. [7] to determine time-averaged velocity fields. An average correlation method is used to improve the signal-to-noise ratio of the correlation function. The measurement depth, beyond which image intensity has only a negligible effect on velocity measurements, is $4.7 \mu \mathrm{m}$.

Figure 2 shows silicon microchannels which are fabricated with integrated aluminum heaters with standard MEMS processing technology. The channels all have hydraulic diameters less than $150 \mu \mathrm{m}$. Power is applied to the microchannel to initiate bubble generation in the channel from heterogeneous nucleation sites.

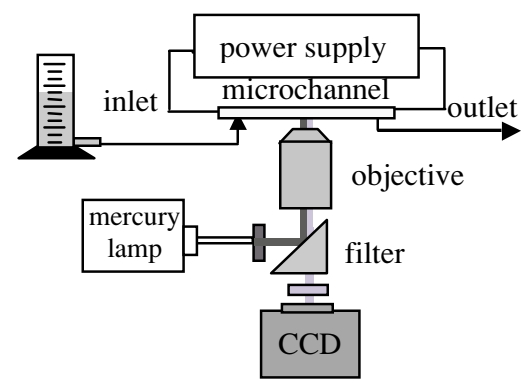

Figure 1. Schematic of $\mu P I V$ imaging system used to image bubble formation and growth in a microchannel .

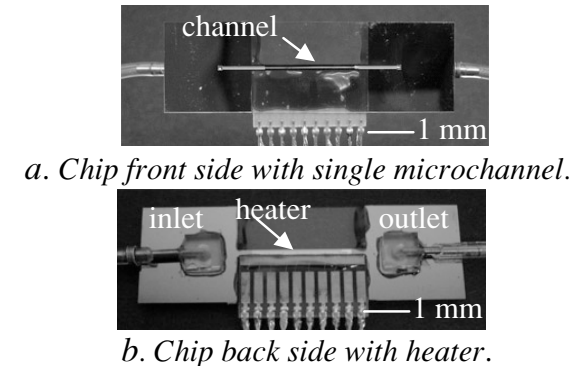

Figure 2. Image of silicon microchannel with integrated sensors and uniform heater.

\section{EXPERIMENTAL RESULTS}

Vapor bubble growth processes were studied under twophase conditions in a microchannel as a function of increasing power. Figure 3 shows images of the growth of a bubble in a hydraulic diameter, $\mathrm{D}_{\mathrm{H}}=140 \mu \mathrm{m}$ channel. Surface tension forces dominate in this Stokes flow regime, $\left(\operatorname{Re}_{\mathrm{D}}=0.02\right)$, where the bubbles do not depart from the walls. The intensity of the images near the liquid-vapor interface is higher due to diffraction and potential particle coalescence as the vapor bubble grows. 


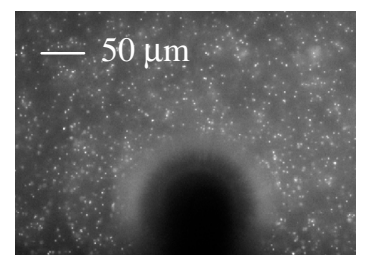

(a) $P=0.98 \mathrm{~W}$

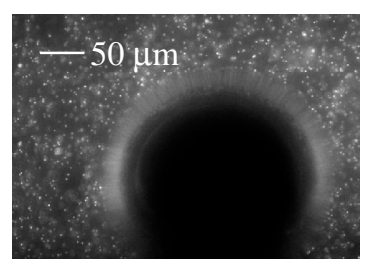

(b) $P=1.4 \mathrm{~W}, 13.8$ s later

Figure 3. Images of bubble growth in a $D_{H}=140 \mu \mathrm{m}$ microchannel with particles seeded in the flow.

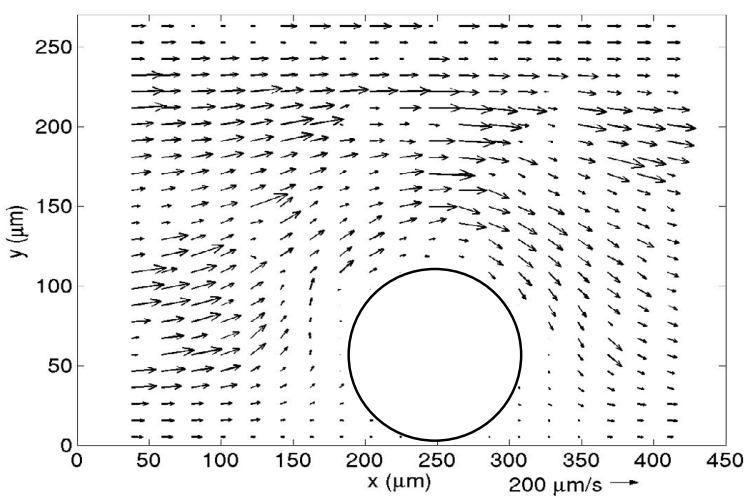

(a) $P=0.98 \mathrm{~W}$

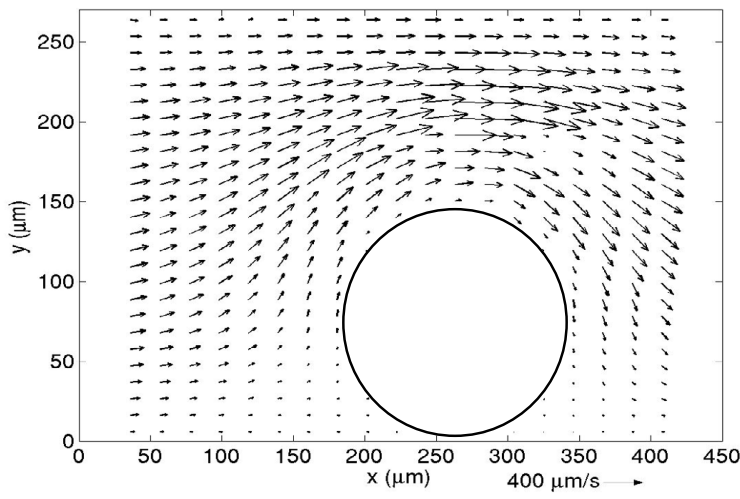

(b) $P=1.4 \mathrm{~W}, 13.8$ s later

Figure 4. Liquid velocity fields in the region around a growing vapor bubble which corresponds to images in Figure 3.

Figure 4 shows velocity fields that correspond to the images of a vapor bubble growing in the microchannel in Figure 3. As the power level increases from $0.98 \mathrm{~W}$ to $1.4 \mathrm{~W}$, the liquid velocity near the stationary bubble increases as the liquid flow area around the bubble is constrained. The maximum observed velocities in this experiment are $250 \mu \mathrm{m} / \mathrm{s}$ and $420 \mu \mathrm{m} / \mathrm{s}$, respectively, corresponding to Figures $6(\mathrm{a})$ and $6(\mathrm{~b})$.

Figure 4 is an example of the transient liquid velocity fields that can be obtained using $\mu$ PIV when bubbles are created by applying heat to the microchannel. Quantitative estimates of viscous stresses on the bubble can be obtained directly from these velocity data, in place of approximate correlations based on integral quantities of idealized conditions such as drag coefficients $[8,9]$.

To proceed further, pressure fields within and outside of the bubble can be found using a momentum balance on control volumes in the bubble region and relations for the surface tension on the free surfaces. In such measurements, we would obtain twodimensional velocity fields at different depths of the bubble, such that the entire three-dimensional flow field can be obtained.
The ultimate goal of this work is determining criterion for bubble departure from the channel wall, once pertinent forces are quantified. These velocity measurements are an important step in this direction and a contribution to understanding bubble dynamics in a microchannel structure.

\section{CONCLUSIONS}

Progress towards understanding important parameters in bubble growth in a microchannel is demonstrated using $\mu$ PIV. Preliminary measurements of liquid velocity fields surrounding vapor bubbles suggest that quantitative values of forces acting on a bubble should be attainable. Further work is required to extract these forces and determine bubble departure criteria. The method should provide an essential tool for a fundamental understanding of bubble growth in many MEMS devices and ultimately aid in the design of optimized microscale structures.

\section{ACKNOWLEDGEMENTS}

The work is supported by MARCO. Travel support has been generously provided by the Transducers Research Foundation and by the DARPA MEMS and DARPA Bio-Flips programs. The project made use of the National Nanofabrication Users Network facilities funded by the National Science Foundation under award number ECS-9731294.

\section{REFERENCES}

1. R. A. Braff, A. L. Gerhardt, M. A. Schmidt, M. L. Gray, and M. Toner, "A Microbubble-Powered Bioparticle Actuator", Hilton Head Solid-State Sensor, Actuator, and Microsystems Workshop, Hilton Head, SC, 2002.

2. A. P. Papavasiliou, A. Pisano, and D. Liepmann, "HighSpeed and Bi-Stable Electrolysis-Bubble Actuated Gate Valves", Transducers, June 10-14, 2001, Munich, Germany, 2001.

3. T. Jun, and C.-J. Kim, "Valveless Pumping Using Traversing Vapor Bubbles in Microchannels", Journal of Applied Physics, 83, 11, 5658-5664 (1998).

4. L. Zhang, J.-M. Koo, L. Jiang, M. Asheghi, K. E. Goodson, J. G. Santiago, and T. W. Kenny, "Measurements and Modeling of Two-Phase Flow in Microchannels with Nearly-Constant Heat Flux Boundary Conditions", Journal of Microelectromechanical Systems, 11, 1, 12 -19 (2002).

5. M. Steinke, and S. G. Kandlikar, "Flow Boiling and Pressure Drop in Parallel Flow Microchannels", $1 s t$ International Conference on Microchannels and Minichannels, April 24-25, 2003, Rochester, New York, 2003.

6. L. Jiang, M. Wong, and Y. Zohar, "Phase Change in Microchannel Heat Sinks with Integrated Temperature Sensors", Journal of Microelectromechanical Systems, 8 , 4, 358-365 (1999).

7. C. D. Meinhart, S. T. Wereley, and J. G. Santiago, "A Piv Algorithm for Estimating Time-Averaged Velocity Fields", Journal of Fluids Engineering, 122, 2, 285-289

8. J. F. Klausner, R. Mei, D. M. Bernhard, and L. Z. Zeng, "Vapor Bubble Departure in Forced Convection Boiling", International Journal of Heat and Mass Transfer, 36, 651-662 (1993).

9. R. Clift, J. R. Grace, and M. E. Weber, Bubbles, Drops and Particles. 1978, New York: Academic Press. 